\title{
PROGRESS TOWARDS A LASER-COOLED CESIUM ATOMIC FOUNTAIN FREQUENCY STANDARD AT NIST, GAITHERSBURG
}

\author{
William M. Klipstein ${ }^{1}$, Christopher R. Ekstrom ${ }^{2}$, Steven L. Rolston', William D. Phillips I, (1) \\ National Institute of Standards and Technology, Gaithersburg, MD (2) United States Naval \\ Observatory, Washington, D.C.
}

\section{Introduction}

We report here on progress towards the realization of an atomic frequency standard using a fountain of lasercooled cesium atoms. We have recently observed Ramsey fringes on the $9.2 \mathrm{GHz}$ clock transition and have begun preliminary work aimed at characterizing our fountain. This paper will describe the apparatus briefly, discuss our current work and near-term directions. We conclude with a brief discussion of a laser system being built to realize two-dimensional Raman cooling or Raman velocity selection for cooling below the single photon recoil limit. This system will be used either in our fountain or in a cold-atom clock to be flown on the International Space Station, a project undertaken in collaboration with the Time and Frequency Division at NIST, Boulder.

\section{Apparatus}

The realization of an atomic frequency standard using a fountain of laser cooled atoms ushered in a new era of cesium clock performance. [1] The success of the first fountain clock prompted laboratories around the world to begin constructing their own fountain clocks and will likely increase the total number of national primary frequency standards. The Laser Cooling and Trapping group at the National Institute of Standards and Technology has a fountain clock effort which is shown schematically in Figure 1. For a review of the development of atomic fountains refer to Ref. [2]. The details of our apparatus follow.

Atoms are collected in a magneto-optic trap (MOT) and laser-cooled at the bottom of the fountain, then launched using a "moving molasses" of two counterpropagating vertical beams while transverse cooling is maintained with the four horizontal trapping beams. After launching, the atoms pass through two microwave cavities located inside the magnetically shielded interaction region. The first cavity delivers a $\pi$ pulse, transferring atoms from $\left|F, m_{F}\right\rangle=14, m>$ to $13, m>$, after which the downward going laser beam is pulsed on to push atoms in $\mathrm{F}=4$ out of the fountain by radiation pressure. This prepares the atoms for the Ramsey interrogation in the upper cavity.
Optical state detection occurs in two regions below the trapping region. In the upper region, atoms fall through a laser tuned to the $4 \Rightarrow 5$ ' cycling transition. Fluorescence from the atoms in $F=4$ is collected with an efficiency estimated to be approximately $10 \%$ and imaged onto a large area photodiode. The lower detection region adds a repumper laser tuned from $3 \Rightarrow 4$ ' to the cycling transition laser for normalizing the fluorescence signal to the total atom number. The photodiodes in the two regions are wired in parallel but with opposite polarities in a single transconductive amplifier.

The longitudinal magnetic field in the interaction region separates out the different $\Delta \mathrm{m}=0$ transitions and maintains a quantization axis. The field comes from a single-winding solenoid with constant pitch of 4.33 turns $/ \mathrm{cm}$. A constant-current supply provides fields ranging from $10-300 \mu \mathrm{T}$.

The two microwave cavities are $\mathrm{TE}_{011}$ rectangular cavities fed by symmetric pairs of wire loops. The clock cavity has a loaded $Q$ of 18,000 ; the state-selection cavity has a loaded $Q$ of 4500 . Waveguide tubes below cut-off prevent microwave leakage out of the cavities, which are integrally connected to the vacuum enclosure comprised of the copper drift tube, the cavities, and then the high vacuum trapping chamber. Both cavities are regulated to $40.5{ }^{\circ} \mathrm{C}$ using heaters connected to the upper and lower flanges of the tower. To prevent large thermal gradients in the tower, the entire tower is heated with warmed air passed through tubing wound around the outside of the magnetic shields and underneath a layer of thermal insulation. DC breaks on the microwave feedthroughs, electrical insulators and a single ground connection prevent thermally driven currents. The pressure in the chamber is roughly $5 \times 10^{-8} \mathrm{~Pa}$.

For stable operation of a fountain clock, it is desirable to avoid the switching magnetic fields associated with periodic loading of a MOT, so the existing fountain FO-1 at LPTF and all intended primary frequency standards load atoms straight into a molasses from a vapor cell. Our fountain was added onto an existing apparatus used for work on optical lattices, with a trapping region loaded from a slowed atomic beam. After loading the MOT, the magnetic field is switched off for a period of polarization gradient cooling in a three dimensional lin $\perp$ lin molasses. 
The experiment uses seven different diode lasers. Two Littrow-style grating stabilized lasers are used for slowing the atomic beam, while a third is tuned to the 3 $\Rightarrow 4^{\prime}$ transition and is used as a repumper for trapping, cooling and detection. A commercial Littman-cavity laser is offset locked to the $4 \Rightarrow 3^{\prime} \times 4^{\prime}$ saturated absorption feature in a vapor cell and serves as a master oscillator for three slave lasers. Part of the master beam passes through a double-pass $80 \mathrm{MHz}$ acousto-optic modulator (AOM) and injects the slave used for the detection beam; part passes through a double pass AOM before injecting the laser used for trapping; and part of the master directly locks a laser used for the launch beams. The launch beam

trapping, launching, cooling, and cleanup laser beams

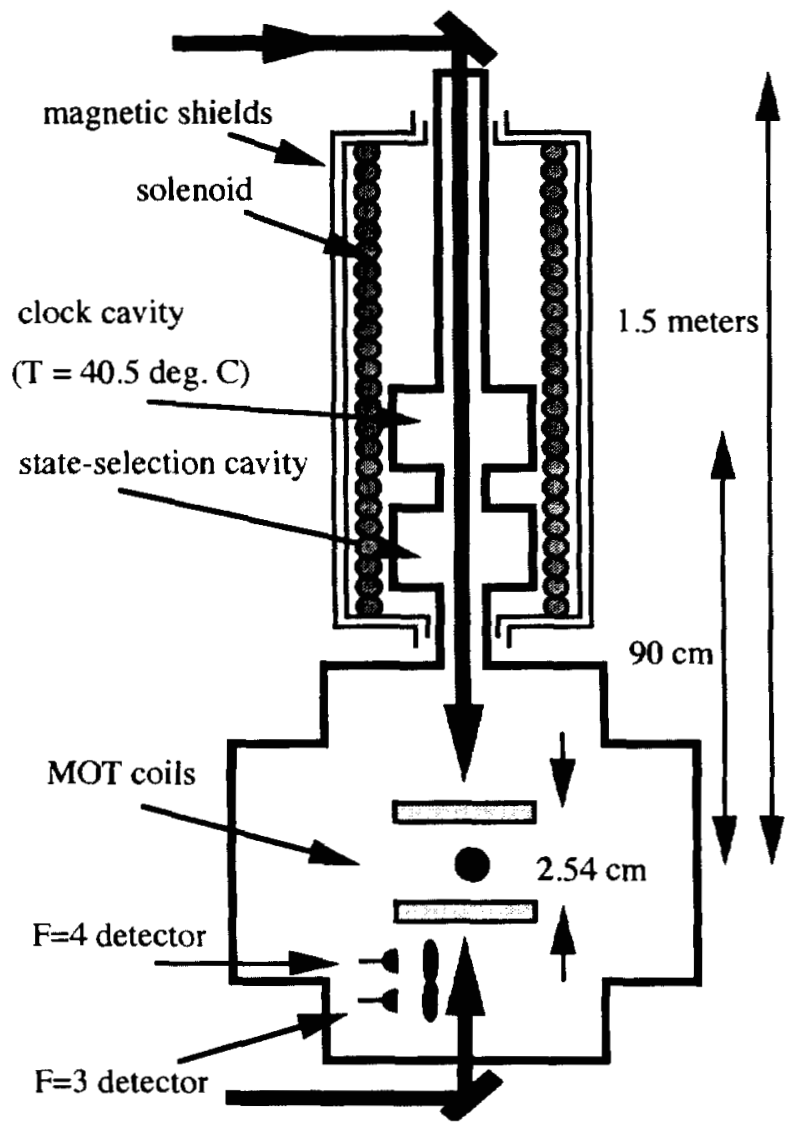

Figure 1. Schematic of apparatus.

is split into two parts each of which goes through a double-pass AOM followed by a polarizing fiber which delivers the light in a clean spatial mode to the atoms.

Since we launch atoms using a three dimensional molasses, meaning the transverse cooling beams are on at the same time as the launch beams, we force the horizontal laser beams have the average frequency of the two launch beams in order to avoid introducing velocity sidebands on the launch. [3] This is achieved by taking the oscillators controlling the launch beam frequencies, mixing them together and taking the sum, then dividing the sum with a flip-flop to produce the average frequency. This signal is then amplified and drives the AOM used to generate the trapping laser frequency. The frequencies used during launch are derived from voltage controlled oscillators; a stable final velocity comes from using preset synthesizers during the final cooling stage.

Table 1. Time sequence of an experimental cycle.

\begin{tabular}{|ll|}
\hline MOT load & $1.0 \mathrm{~s}$ \\
\hline pol. grad. cooling & $5.7 \mathrm{~ms}$ \\
\hline near resonant launch & $1.5 \mathrm{~ms} \quad\left(\mathrm{I} \sim 10 \mathrm{I}_{\text {sat }}\right)$ \\
\hline post cool & $1.8 \mathrm{~ms} \quad$ (detuning $\sim 5.5 \Gamma)$ \\
\hline flight time & $0.9 \mathrm{~s} \quad(0.28 \mathrm{~s}$ Ramsey time) \\
\hline detection window & $50 \mathrm{~ms}$ \\
\hline
\end{tabular}

\section{Observation of Resonance Spectra}

All of the individual systems required for clock operation are in place and operational, if not optimized. The time sequence for a single experimental cycle is summarized in Table 1. Figure 2 shows Ramsey fringes on the clock transition, showing a FWHM of $1.8 \mathrm{~Hz}$. Each point reflects a single trap load and launch through the microwave cavities. The fluorescence time of flight signal from only one detection region is fitted to a Maxwell-Boltzmann distribution; the amplitude from the fit is plotted. This data is unnormalized to total atom number and does not take into account the width of the distribution, which would only require a simple software change which has since been implemented.

Also shown in Fig. 2 is a close up of the central fringe, along with a fit to sine squared. The $5 \mathrm{mHz}$ frequency uncertainty in the fit for these 55 points corresponds to a short term stability of $6 \times 10^{-12} \tau^{-1 / 2}$. This estimate is overly conservative, as the contribution to the frequency uncertainty comes almost entirely from the fraction of points on the steep part of the curve. We would like to be limited by our local oscillator, which was built by Fred Walls of NIST/Boulder and should allow us to achieve a fractional short term stability of approximately $2 \times 10^{-13}$ at one second.

The lack of normalization is believed to be the limiting noise source in the data shown. We currently run with roughly $6 \%$ fluctuation in the number of atoms loaded from one cycle to the next. Preliminary subsequent data taken using both detection zones showed an immediate albeit modest improvement of a factor of 2 reduction in noise.

We estimate the number of detected atoms to be $2 \mathrm{x}$ $10^{5}$, which we deduce both from the photocurrent and from the fraction of atoms we expect to return to the bottom of the fountain given a launch temperature of approximately $10 \mu \mathrm{K}$.

Figure 3 shows Ramsey fringes on the $m=1$ and $m=3$ magnetically sensitive transitions in absolute frequency units for a magnetic field of $1.4 \mu \mathrm{T}$. For these fringes, 
the $5 \mathrm{MHz}$ base of our frequency chain was phase locked to the $5 \mathrm{MHz}$ output of an HP5061 clock. These fringes were recorded just after our first observation of Ramsey fringes and show an extremely low signal-to-noise ratio. Nevertheless, these fringes allow us to make a preliminary calibration of our magnetic field winding, and the fact that we have fringe contrast on the $m=3$ transition allows us to set an upper limit on the transverse magnetic field gradient
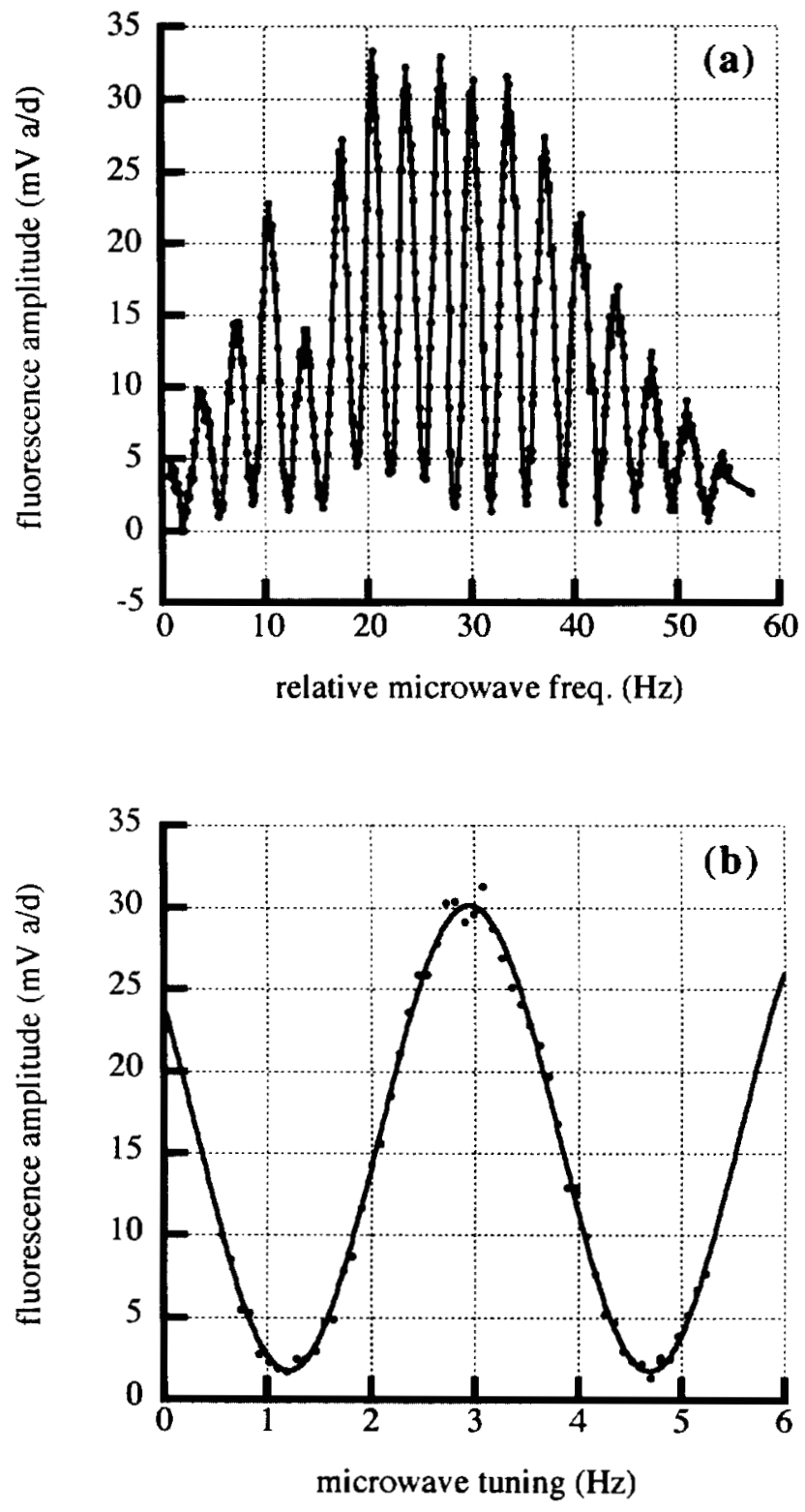

Figure 2. Ramsey fringes on the clock transition. (a) Fluorescence of $\mathrm{F}=4$ atoms in only one detection region, unnormalized for atom number. The dip near $15 \mathrm{~Hz}$ results from the repumper laser drifting beyond its lock range, causing fewer atoms to be collected. (b) Blow up of data from the central fringe, with a fit to sine squared. Fringe width is $1.8 \mathrm{~Hz}$, uncertainty in line center is 5 $\mathrm{mHz}$. averaged over the atomic trajectories of $<80 \mathrm{pT} / \mathrm{cm}$.

We have observed Ramsey fringes in our fountain with a FWHM below $1 \mathrm{~Hz}$, but these correspond to launches outside of the inner magnetic shield and so will not be useful for any measurements. We should be able to have a fringe width of $1 \mathrm{~Hz}$ with our geometry, although we have not yet mapped out the longitudinal variation of the $\mathrm{C}$ field to verify that the field remains flat and stable for such a launch height.

\section{Laser system for cooling below the single-photon recoil limit}

In an atomic fountain the line $Q$ is limited by the interaction time, which is determined by the time of flight above the cavity and hence the pull of gravity. Constructing a fountain with a $1 \mathrm{~Hz}$ linewidth requires the construction of a fountain at least $1.25 \mathrm{~m}$ high. A further narrowing of this line by even a factor of 3 would require a tower over 11 meters high, prohibitive in a laboratory setting. A cold atom clock in space, however, would allow a greater interaction time, and so NIST has undertaken the construction of a cold-atom clock for the International Space Station.

A cesium fountain running with a 1 second flight time will lose roughly $75 \%$ of the atoms cooled to a few $\mu \mathrm{K}$ due to the residual transverse velocity and the size of the aperture in the microwave cavity. Extending the interaction time exacerbates this problem, reducing the fraction of atoms contributing useful signal while still contributing to the cold-collisional frequency shift [4]. In order to realize the highest performance from the space clock we are interested in reducing the transverse velocity of the atoms to $0.3 v_{\text {recoil }}$, which for cesium corresponds to $1 \mathrm{~mm} / \mathrm{s}$ RMS velocity. We intend to realize this cooling using either two-dimensional Raman velocity selection [5], if we can afford to throw away atoms, or two-dimensional Raman cooling [6] if we need to maintain atomic flux.

In support of this project, our group is constructing a laser system to enable us to realize velocity-selective twophoton transitions between the two hyperfine levels. The system again makes use of a master laser and two slaves. The master is a $150 \mathrm{~mW}$ DBR laser with a linewidth of 5 MHz. A $5 \mathrm{~mW}$ beam from this master laser is coupled via polarizing fiber to an electro-optic modulator operating at $4.6 \mathrm{GHz}$, half the cesium hyperfine frequency. When driven with around $200 \mathrm{~mW}$ RF power, this modulator should give $20 \%$ of the optical power in each sideband and suppress the carrier. We intend to inject all optical frequencies into each slave, then lock the slave to a particular line by adjusting the current of each laser and even to switch lines by changing the currents. We are currently setting up the injection of the slaves to test the time response of this system before developing the Raman capabilities. A near-identical system has been developed at LPTF and described in Ref. [7]. 


\section{Conclusion}

We have recently observed Ramsey fringes in our atomic fountain and continue to optimize the signal and characterize the stability and sensitivity of the device. We have begun development of a laser system with the purpose of realizing two dimensional cooling below the single-photon recoil limit.
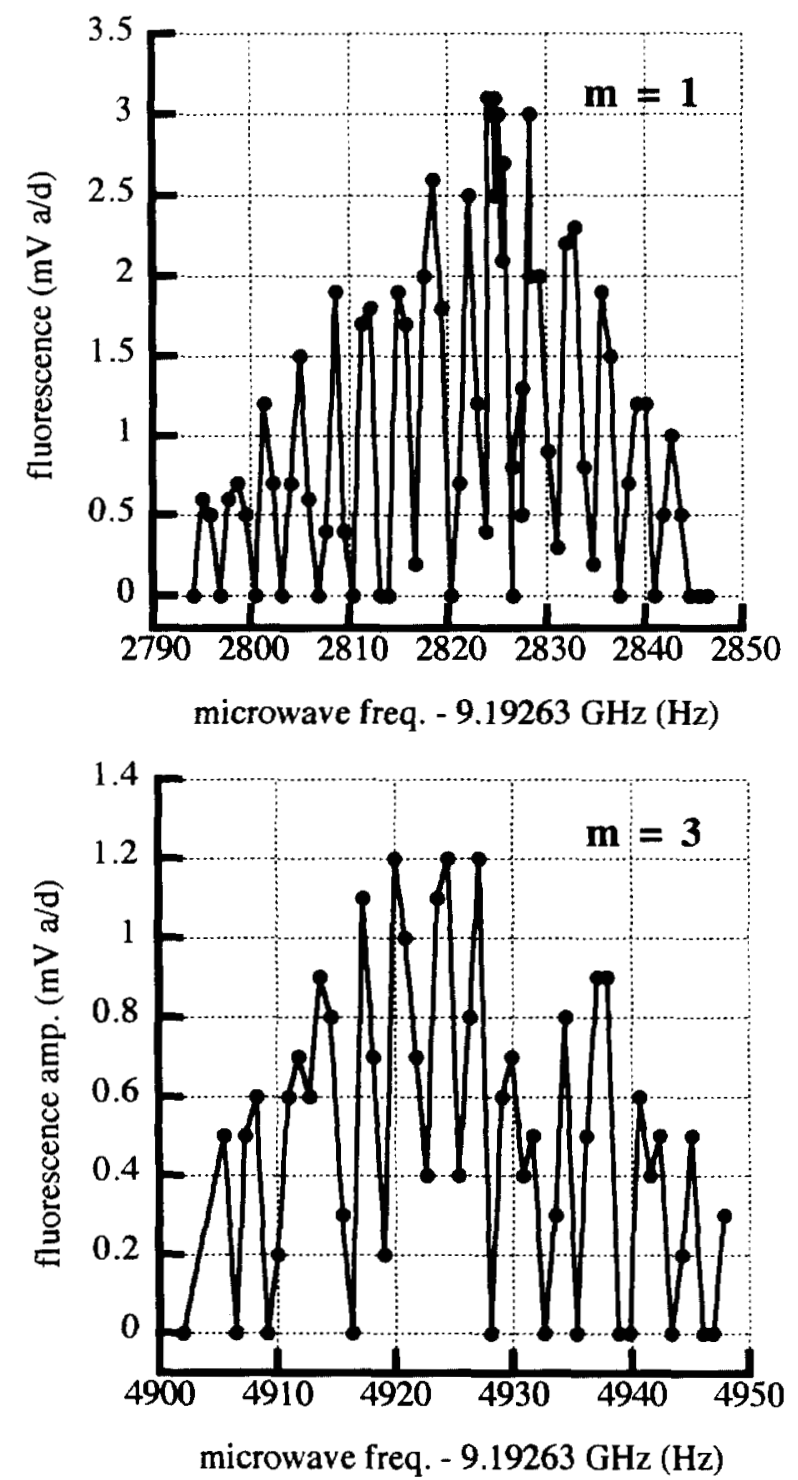

Figure 3. Ramsey fringes on magnetically sensitive transitions in absolute frequency units for a magnetic field of $0.14 \mu \mathrm{T}$. (a) Fringes for transitions between $\mathrm{m}=1$ levels. (b) Fringes for transitions between $m=3$ levels. Despite the noisy signal, the contrast in these fringes sets an upper limit on the size of transverse magnetic field gradients of $80 \mathrm{pT} / \mathrm{cm}$.

\section{References}

[1] Ghezali, S., Laurent, Ph., Lea, S.N., and Clairon, A., "An experimental study of the spin-exchange frequency shift in a laser-cooled cesium fountain frequency standard, "Europhys. Lett. 36 (1996) 25. ; A. Clairon, S. Ghezali, G. Santarelli, Ph. and K. Szymaniec, 1995, "Preliminary Accuracy Evaluation of a Cesium Fountain Frequency Standard," Proceedings of the Fifth Symposium on Frequency Standards and Metrology, p. 49-59 (Woods Hole, MA); A. Clairon, Bahoura, 1995, "A Cesium Fountain Frequency Standard: Preliminary Results," IEEE Trans. Instrum. Meas. 44, p. 128-1313.

[2] Weiss DS, Young BC, Chu S, "PrecisionMeasurement Of H/M(Cs) Based On Photon Recoil Interferometry," App. Phys. B-Las. and Opt. 59, p. 217-256 (1994); Gibble, K., Chang, S. and Legere, R., "Direct Observation of S-Wave Atomic Collisions," Phys. Rev. Lett., 75 p. 2666 (1995); Ohshima S, Kurosu T, Ikegami T, Nakadan Y, "Cesium Atomic Fountain With 2-Dimensional Moving Molasses," Jap. J. of Ap. Phys. - 2 34, p. L1170-L1173 (1995); Featonby PD, Webb CL, Summy GS, Foot CJ, Burnett K, "Observation of light-induced coherence loss in a caesium atomic fountain," J. Phys. B-At. Mol. and Opt. Phys. 31, p. $375-381$ (1998).

[3] Selma Ghezali, PhD thesis, LPTF, Paris (1998).

[4] Gibble K, Chu S, "Laser-Cooled Cs Frequency Standard and a Measurement of the Frequency-Shift Due To Ultracold Collisions" Phys. Rev. Lett. 70, p. 1771-1774 (1993)

[5] M. Kasevich et al., "Atomic Velocity Selection Using Stimulated Raman Transitions," Phys. Rev. Lett., 66 (1991) 2297.

[6] Davidson N, Lee HJ, Kasevich M, Chu S, "Raman Cooling of Atoms In 2-Dimensions And 3Dimensions," Phys Rev. Lett. 72, p. 3158-3161 (1994); Lee HJ, Adams CS, Kasevich M, Chu S, "Raman cooling of atoms in an optical dipole trap," Phys. Rev. Lett 76 p. 2658-2661 (1996); Reichel j, et al., "Cooling of Cesium Below $3 \mathrm{nK}$ - New Approach Inspired by Levy Flight Statistics, Phys. Rev. Lett. 75, p. 4575-4578 (1995).

[7] Szymaniec K, Ghezali S, Cognet L, Clairon, A, "Injection locking of diode lasers to frequency modulated source," Opt. Comm. 144, p. 50-54 (1997). 